\title{
Bounds on the Spectral Shift Function and the Density of States ${ }^{\star}$
}

\author{
Dirk Hundertmark $^{1}$, Rowan Killip ${ }^{2}$, Shu Nakamura ${ }^{3}$, Peter Stollmann ${ }^{4}$ \\ Ivan Veselic ${ }^{4}$
}

1 Department of Mathematics, University of Illinois at Urbana-Champaign, Illinois 61801, USA. E-mail: dirk@math.uiuc.edu

2 Mathematics Department, UCLA, Los Angeles, CA 90095-1555, USA. E-mail: killip@ math.ucla.edu

3 Graduate School of Mathematical Science, University of Tokyo, 3-8-1 Komaba, Meguro Tokyo, 153-8914 Japan. E-mail: shu@ms.u-tokyo.ac.jp

4 Fakultät für Mathematik, Technische Universität Chemnitz, 09107 Chemnitz, Germany.

E-mail: P.Stollmann@mathematik.tu-chemnitz.de; ivan.veselic@mathematik.tu-chemnitz.de

\begin{abstract}
We study spectra of Schrödinger operators on $\mathbb{R}^{d}$. First we consider a pair of operators which differ by a compactly supported potential, as well as the corresponding semigroups. We prove almost exponential decay of the singular values $\mu_{n}$ of the difference of the semigroups as $n \rightarrow \infty$ and deduce bounds on the spectral shift function of the pair of operators.

Thereafter we consider alloy type random Schrödinger operators. The single site potential $u$ is assumed to be non-negative and of compact support. The distributions of the random coupling constants are assumed to be Hölder continuous. Based on the estimates for the spectral shift function, we prove a Wegner estimate which implies Hölder continuity of the integrated density of states.
\end{abstract}

\section{Introduction and Results}

In this paper we analyze the spectral properties of multi-dimensional Schrödinger operators. First, we consider a pair of operators $H_{1}, H_{2}$ which differ by a compactly supported potential $u$. The singular values $\mu_{n}$ of the difference of the corresponding exponentials $V_{\text {eff }}:=e^{-H_{1}}-e^{-H_{2}}$ are shown to decay almost exponentially in $n$.

This result allows us to deduce a bound on the Lifshitz-Krein spectral shift function (SSF) of the operator pair $H_{1}, H_{2}$. We give a bound on the SSF when integrated over the energy axis against a bounded, compactly supported function. In turn, the bound on the SSF is used to prove a Wegner estimate for random Schrödinger operators of alloy type and Hölder continuity of the integrated density of states (IDS). Our estimates have a better continuity in the energy parameter than previously known bounds. Moreover, we are able to treat random coupling constants, whose distribution does not have

* (c) 2005 by the authors. Faithful reproduction of this article, in its entirety, by any means is permitted for non-commercial purposes. 
a density. In particular, for Hölder continuous distributions we prove that the IDS is Hölder continuous, too.

We will treat magnetic Schrödinger operators

$$
H=H_{A}+V=(-i \nabla-A)^{2}+V
$$

acting on $\mathbb{R}^{d}$ whose potentials (magnetic and electric) obey the following hypotheses: Each component of $A$ is $L_{\text {loc }}^{2}$. The positive part of the electric potential, $V_{+}:=$ $\max (0, V)$, belongs to $L_{\text {loc }}^{1}$ and the negative part, $V_{-}:=\max (0,-V)$, is in the Kato class. Notice that under our convention, $V=V_{+}-V_{-}$.

For a general discussion of the Kato-class, see [15]; for its relevance to the Feynman-Kac formula see, e.g., [2, 6, 49]. In particular, $V_{-}$is in the Kato-class, if

$$
\left\|V_{-}\right\|_{L_{\text {loc,unif }}^{p}\left(\mathbb{R}^{d}\right)}=\sup _{x \in \mathbb{R}^{d}}\left(\int_{|x-y| \leq 1}\left|V_{-}(y) d y\right|\right)^{1 / p}<\infty,
$$

where $p=1$ if $d=1$ and $p>d / 2$ if $d \geq 2$. Thus the allowed potentials cover all physically relevant cases.

Under these hypotheses, one may define $H$ via the corresponding quadratic form (with core $C_{c}^{\infty}$ ). By the same method, one can define the Dirichlet restriction of $H$ to the cube $\Lambda_{l}=[-l / 2, l / 2]^{d}, l \geq 1$. This will be denoted $H^{l}$.

Let $H_{1}$ be a Schrödinger operator of the form just described and let $H_{2}=H_{1}+u$, where $u=u_{+}-u_{-}$obeys the hypotheses for electric potentials just described.

The starting point for our analysis is an estimate on the singular values of $V_{\text {eff }}:=$ $e^{-H_{1}}-e^{-H_{2}}$ and on the corresponding object in the finite volume case, namely $V_{\mathrm{eff}}^{l}:=$ $e^{-H_{1}^{l}}-e^{-H_{2}^{l}}$. Recall that the singular values of a compact operator $A$ are the squareroots of the eigenvalues of $A^{*} A$. We will enumerate them as $\mu_{1}(A) \geq \mu_{2}(A) \geq \cdots \geq 0$ according to multiplicity.

Theorem 1. There are finite positive constants $c$ and $C$ such that the singular values of the operator $V_{\text {eff }}^{l}$ obey

$$
\mu_{n} \leq C e^{-c n^{1 / d}}
$$

In fact, $c$ may be chosen depending only on the dimension, while $C$ depends on the Kato-class norms of $u_{-}, V_{-}$and on the diameter of the support of $u_{+}$.

The same estimate holds for the singular values of $V_{\mathrm{eff}}$.

Remarks. i) In particular $\left\|V_{\text {eff }}^{l}\right\|_{\mathcal{J}_{p}}:=\sum_{n} \mu_{n}^{p}$ is finite and thus $V_{\text {eff }}^{l}$ is an element of the operator ideal $\mathcal{J}_{p}:=\left\{A\right.$ compact $\left.\mid\|A\|_{\mathcal{J}_{p}}<\infty\right\}$ for any $p>0$. Thus our result can be understood as a sharpening and generalization of norm, Hilbert Schmidt, and trace bounds on the difference of semigroups, derived e.g. in [7-9, 16-19, 48, 51, 52].

ii) Note that the estimate (2) depends on the positive part of $u$ only through supp $u$. Thus, for $u=\lambda \tilde{u}$, where $\tilde{u} \geq 0$ and $\lambda$ is a non-negative coupling constant, the estimate is independent of the choice of $\lambda$. Moreover

(1) $u$ may be taken to $+\infty$ on its support. In this case $H_{2}$ equals the restriction of $H_{1}$ to $\mathbb{R}^{d} \backslash$ supp $u$ with Dirichlet boundary conditions, provided the boundary of supp $u$ obeys some mild regularity conditions; see, e.g., [53]. 
(2) Similarly, $H_{1}$ may be defined on a set strictly smaller than $\mathbb{R}^{d}$ : Let $D \subset \mathbb{R}^{d}$ be open, $H_{A}^{D}$ the Dirichlet restriction of $H_{A}$ on $D$, and $H_{1}=H_{A}^{D}+V$, where $V$ satisfies the same conditions as before. In this case $H_{j}^{l}$ is the Dirichlet restriction of $H_{j}, j=1,2$ to the set $\Lambda_{l} \cap D$.

iii) The proof of Theorem 1 is surprisingly simple. Morally, the result is an immediate consequence of Weyl's law for the eigenvalue asymptotic of Dirichlet Laplacians on compact domains. This suggests that the decay rate of the singular values of $e^{-H_{1}}-e^{-H_{2}}$ is, in fact, given by $\exp \left(-c n^{2 / d}\right)$. One might ask, however, whether the singular values could not typically decay at a much faster rate. It turns out that a decay rate like $\exp \left(-c n^{\alpha}\right)$ for $\alpha>2 / d$ is impossible, see Remark ii) after Theorem 2 below. This leaves open the cases $\alpha \in(1 / d, 2 / d]$. We conjecture that, in fact, the true bound is of the form $\mu_{n} \leq C \exp \left(-c n^{2 / d}\right)$.

Our interest in Theorem 1 comes from the fact that it allows us to derive an integral bound on the the SSF $\xi\left(\lambda, H_{2}, H_{1}\right)$ of the pair of operators $H_{1}, H_{2}$, which shows that the SSF can have only very mild local singularities. (See Sect. 2.1 for a precise definition of the SSF.) The SSF plays a role in different areas of mathematical physics, for instance in scattering theory, cf. e.g. [59], and the study of surface potentials, see [10,36]. Various of its properties are discussed in the literature: monotonicity and concavity in [20, 22, 35], the asymptotic behaviour in the large coupling constant [43, 46, 44] and semiclassical limit $[42,40]$. See $[5,34]$ for surveys.

For $t>0$ let $F_{t}:[0, \infty) \rightarrow[0, \infty)$ be defined by

$$
F_{t}(x)=\int_{0}^{x}\left(\exp \left(t y^{1 / d}\right)-1\right) d y .
$$

As the integrand is increasing, $F_{t}$ is a convex function.

Theorem 2. Let $\xi$ be the spectral shift function for the pair $H_{1}, H_{2}$ or $H_{1}^{l}, H_{2}^{l}$.

i) Let $F_{t}$ be defined as above. There exisits a constant $K_{1}$, depending on $t$, such that for small enough $t>0$,

$$
\int_{-\infty}^{T} F_{t}(|\xi(\lambda)|) d \lambda \leq K_{1} e^{T}<\infty
$$

for all $T<\infty$.

ii) There exist constants $K_{1}, K_{2}$ depending only ond, diam supp $u_{+}$and the Kato class norms of $V_{-}, u_{-}$, such that for any bounded compactly supported function $f$,

$$
\int f(\lambda) \xi(\lambda) d \lambda \leq K_{1} e^{b}+K_{2}\left\{\log \left(1+\|f\|_{\infty}\right)\right\}^{d}\|f\|_{1}
$$

with $b=\sup \operatorname{supp}(f)$.

Remarks. i) Note that the function $F_{t}$ defined in (3) has the asymptotic behavior

$$
F_{t}(x) \sim d x^{(d-1) / d} \exp \left(t x^{1 / d}\right) \quad \text { for large } x .
$$

Thus, by part i) of Theorem 2, the spectral shift function can have at most mild logarithmic local singularities. It is tempting to think that, at least for non-negative compactly supported perturbations, the spectral shift function should always be 
bounded. However, this is not the case. For a perturbation of the free Schrödinger operator with a constant magnetic field by a compactly supported potential, Raikov and Warzel showed that the spectral shift function diverges at each Landau level $E_{q}$ like

$$
\left|\xi\left(E_{q}+\lambda\right)\right| \sim\left(\frac{|\ln (\lambda)|}{\ln |\ln \lambda|}\right)^{d / 2} \quad \text { as } \lambda \downarrow 0
$$

see [45] for the case $d=2$ and [39] for the generalization to even dimensions. Thus, setting $F_{t, \alpha}(x):=\int_{0}^{x}\left(\exp \left(t y^{\alpha}\right)-1\right) d y$, the asymptotic (6) implies that $F_{t, \alpha}(|\xi|)$ is locally integrable if and only if $0 \leq \alpha \leq 2 / d$, whereas Theorem 2 guarantees it only for $\alpha \leq 1 / d$ (and $t$ small enough, if $\alpha=1 / d$ ).

ii) The proof of Theorem 2 shows that if Theorem 1 holds in the form $\mu_{n} \leq c_{1}$ $\exp \left(-c_{2} n^{\alpha}\right)$, then $F_{t, \alpha}(|\xi|) \in L^{1}(-\infty, T)$ for small enough $t$ (and all finite $T$ ). Thus the Raikov-Warzel result shows that the estimate in Theorem 1 cannot be improved beyond $C \exp \left(-c n^{2 / d}\right)$.

iii) An example without magnetic fields, where the SSF shows unexpected divergencies, was studied by Kirsch in [28, 29]. It is related to the one with a constant magnetic field in that the high degeneracy of eigenvalues plays a crucial role. Let $E>0, u$ non-negative, bounded with compact support, and not identically equal to zero, $a:[0, \infty) \rightarrow(0, \infty)$, and $\xi_{l}(\cdot):=\xi\left(\cdot,-\Delta^{l},(-\Delta+a(l) u)^{l}\right)$. Then $\limsup _{l \rightarrow \infty} \xi_{l}(E)=\infty$, for any $E, a$ and $u$ as above. This result relies on the degeneracy of eigenvalues of the pure Dirichlet Laplacian on a cube. There is, however, a set of full measure $\mathcal{E} \subset \mathbb{R}$ with dense complement such that $\lim _{\mathbb{N} \ni l \rightarrow \infty} \xi_{l}(E)=0$, for all $E \in \mathcal{E}$, if $a(l) \leq l^{-k}, k>3$.

iv) In contrast to the above unboundedness results, Sobolev, [50] showed that for the pair $H_{1}=-\Delta$ and $H_{2}=-\Delta+u$ with $|u(x)| \leq$ const. $(1+|x|)^{-\alpha}$ and $\alpha>d$, the spectral shift function $\xi$ is, indeed, locally bounded. However, this type of result seems to require very strong hypotheses on $H_{1}$, for example, a trace class limiting absorption principle and in particular, that $H_{1}$ has absolutely continuous spectrum on the positive real axis.

Theorem 2.ii) has a nice consequence in the theory of random Schrödinger operators. In this case, we take $f$ to be the derivative of a smooth, monotone switch function $\rho:=\rho_{E, \varepsilon}: \mathbb{R} \rightarrow[-1,0]$. By a switch function we mean that for a positive $\varepsilon \leq 1 / 2$ it has the following properties: $\rho \equiv-1$ on $(-\infty, E-\varepsilon], \rho \equiv 0$ on $[E+\varepsilon, \infty)$ and $\left\|\rho^{\prime}\right\|_{\infty} \leq 1 / \varepsilon$. Theorem 2.ii) and the Krein trace identity, see $\S 2.1$, then imply that there is a constant $C_{E}$ such that

$$
\operatorname{Tr}\left[\rho\left(H_{2}\right)-\rho\left(H_{1}\right)\right] \leq C_{E}|\log (\varepsilon)|^{d} .
$$

The estimate (7) improves upon the bound derived by Combes, Hislop and Nakamura in [14]. They prove that for any exponent $\alpha<1$, there is a constant $\tilde{C}_{E}(\alpha)$ depending only on $d, C_{0}$, diam $\operatorname{supp} u, E+\varepsilon$ and $\alpha$ such that

$$
\operatorname{Tr}\left[\rho\left(H_{2}\right)-\rho\left(H_{1}\right)\right] \leq \tilde{C}_{E}(\alpha) \varepsilon^{-\alpha} .
$$

An alloy type model is a random Schrödinger operator $H_{\omega}=H_{0}+V_{\omega}$, where $H_{0}=H_{A}+V_{\text {per }}$ with a periodic potential $V_{\text {per }}$. The random part of the potential has the form $V_{\omega}(x)=\sum_{k \in \mathbb{Z}^{d}} \omega_{k} u(x-k)$. The coupling constants $\omega_{k}, k \in \mathbb{Z}^{d}$, are a sequence of bounded random variables, which are independent and identically distributed with 
distribution $\mu$. The expectation of the product measure $\bigotimes_{k \in \mathbb{Z}^{d}} \mu$ is denoted by $\mathbb{E}$. The single site potential $u \neq \equiv 0$ is of compact support. Denote for $\varepsilon>0$,

$$
s(\mu, \varepsilon)=\sup \{\mu([E-\varepsilon, E+\varepsilon]) \mid E \in \mathbb{R}\} .
$$

With this definition, we have

Theorem 3. Let $H_{\omega}$ be an alloy type model and $u \geq \kappa \chi_{[-1 / 2,1 / 2]^{d}}$ for some positive $\kappa$. Then for each $E_{0} \in \mathbb{R}$ there exists a constant $C_{W}$ such that, for all $E \leq E_{0}$ and $\varepsilon \leq 1 / 2$,

$$
\mathbb{E}\left\{\operatorname{Tr}\left[\chi_{[E-\varepsilon, E+\varepsilon]}\left(H_{\omega}^{l}\right)\right]\right\} \leq C_{W} s(\mu, \varepsilon)\left(\log \frac{1}{\varepsilon}\right)^{d}\left|\Lambda_{l}\right| .
$$

In particular, if $\mu$ is Hölder continuous with exponent $\alpha$, then the $\varepsilon$-dependence of the RHS of (10) is $\varepsilon^{\alpha}\left(\log \frac{1}{\varepsilon}\right)^{d}$. In [54], Stollmann proved a weaker version of (10) with RHS equal to $C_{W} s(\mu, \varepsilon)\left|\Lambda_{l}\right|^{2}$.

Bounds like (10) are called Wegner estimates. They were first deduced by physical reasoning by Wegner in [58] for the Anderson model, the finite difference analogue of the alloy type model. Wegner estimates are important $a$ priori estimates, used to derive regularity properties of the integrated density of states (IDS) and to prove localization for random Schrödinger operators. In this context, localization means the existence of an energy region where the random operator has almost surely dense pure point spectrum with exponentially decaying eigenfunctions. See, e.g., [55] for a monograph exposition and, e.g., [21] (and the references therein) for more recent developments.

The proof of Theorem 3 can be directly applied to Anderson type models, i.e. random Schrödinger operators on $\ell^{2}\left(\mathbb{Z}^{d}\right)$. In this case, a compactly supported potential is a finite rank operator. For such perturbations the supremum-norm of the induced SSF is bounded by the rank of the operator, see e.g. [5, 14]. The uniform bound on the SSF yields a bound like (10) with the RHS side equal to $C_{W} s(\mu, \varepsilon)|\Lambda|$ and the constant $C_{W}$ independent of the energy $E$. This gives an easy proof of a Wegner estimate for Hölder continuous single site distributions $\mu$.

The IDS $N(E)$ is defined as the limit of the distribution functions,

$$
N_{\omega}^{l}(E):=\left|\Lambda_{l}\right|^{-1} \#\left\{\text { eigenvalues of } H_{\omega}^{l} \text { not greater than } E\right\},
$$

as $l$ tends to infinity. For almost all $\omega \in \Omega$ the limit exists and is independent of $\omega$. As a consequence of Theorem 3, the IDS of the above alloy-type model satisfies

$$
\left|N\left(E_{1}\right)-N\left(E_{2}\right)\right| \leq C_{I} s\left(\mu,\left|E_{1}-E_{2}\right|\right)\left(\log \frac{1}{\left|E_{1}-E_{2}\right|}\right)^{d}, \quad\left|E_{1}-E_{2}\right| \leq 1 / 2,
$$

where the constant $C_{I}$ may be chosen uniformly if $E_{1}$ and $E_{2}$ vary in a compact interval $I$. Note that this continuity result cannot be obtained from a Wegner estimate with quadratic dependence on the volume of the box $\Lambda_{l}$. It also shows that, up to a logarithmic correction, the IDS enjoys the same regularity properties as the distribution of the random potential.

Let us return to the discussion of the regularity of the SSF. Denote by $H_{1}=H_{\omega}-\omega_{0} u$ and $H_{2}=H_{1}+u$ the alloy type operators where the value of the coupling constant at $k=0$ is frozen and equal to 0 and $\omega_{0}$ respectively. The other coupling constants are still random. Despite the examples given above it is still possible that the average of the SSF $\bar{\xi}(\lambda):=\mathbb{E}\left\{\xi\left(\lambda, H_{1}, H_{2}\right)\right\}$ over the random background environment is locally bounded. This then implies no logarithmic loss in the Wegner estimate, and thus the Lipschitz continuity of the integrated density of states. 
Indeed, such a bound on $\bar{\xi}$ for $d \leq 3$ has recently been announced by Combes and Hislop, [23]. They have to assume that the single site distribution has a bounded density with respect to Lebesgue measure. So far, the averaging techniques at our disposal do not seem to be sufficient enough to prove that $\bar{\xi}(\lambda)$ is locally bounded for rough single site distributions, even if they are Hölder continuous.

In this context we would like to mention bounds on averaged fractional powers of the SSF derived in [1].

As a final remark, we discuss how Theorem 2 can be used to improve Wegner estimates for alloy type models with somewhat different properties than in Theorem 3. First we present an improvement of a recent Wegner estimate by Combes, Hislop and Klopp [12] for single site potentials with small support.

Theorem 4. Let $H_{\omega}$ be an alloy type model as defined in the paragraph following (8). Assume that $V_{\text {per }}$ has the unique continuation property and is bounded below, $\omega$ is distributed according to a bounded density and $0 \leq u \in L^{\infty}$ is strictly positive on an open set. Then for each $E_{0} \in \mathbb{R}$ there exists a constant $C_{W}$ such that

$$
\mathbb{E}\left\{\operatorname{Tr} \chi_{[E-\varepsilon, E+\varepsilon]}\left(H_{\omega}^{l}\right)\right\} \leq C_{W} \varepsilon\left(\log \frac{1}{\varepsilon}\right)^{d}|\Lambda|
$$

for all $E \leq E_{0}$ and $\varepsilon \leq 1 / 2$.

This follows directly if one uses Theorem 2 instead of the $L^{p}$-estimates on the SSF in the Appendix of [12].

We mention three more disorder regimes where Theorem 2 may be used to simplify proofs of earlier Wegner estimates and to improve the dependence of the estimate in the energy interval length.

(1) Single site potentials with small support and singular coupling constants. Using the perturbation technique of Kirsch, Stollmann and Stolz in [32], we can extend the result from Theorem 3 to single site potentials $u \geq \kappa \chi_{[-s, s]^{d}}$ for some $\kappa, s>0$ in the case of zero magnetic field for energies near spectral edges. In this case no assumption on the unique continuation property is needed.

(2) Coupling constants whose distribution is continuous merely at the extreme values. In [33], Kirsch and Veselić prove a Wegner estimate for alloy type potentials with non-positive single site potentials and coupling constants which have merely in a neighborhood of their maximal value a continuous distribution with bounded density. The estimate applies to energies at the bottom of the spectrum. Using Theorem 2 in the present paper, one can improve the Wegner estimate in [33].

(3) Single site potentials with changing sign. In [24], Hislop and Klopp studied alloy type models with continuous, compactly supported single site potentials, which may take values with both signs, and bounded coupling constants which are distributed according to a bounded, piecewise absolutely continuous density. They prove a Wegner estimate which is Hölder continuous in the energy variable and applies to energies below the spectrum of the non-random, unperturbed operator $H_{0}$. The result extends to internal spectral boundaries in the weak disorder regime. An extension of Theorem 2 in the present paper to the case where the perturbation is equal to a potential sandwiched between the square roots of the resolvent would make it possible to improve the Wegner estimate in [24] with regards to the energy interval length. 
Further results on Wegner estimates for alloy type Schrödinger operators can be inferred from $[4,13,27,30,31,36,56,57]$ and the references therein. Let us mention specifically, that if $\mu$ has bounded density and $u \geq \kappa \chi_{\Lambda_{1}}$ the IDS is actually Lipschitzcontinuous, see [37, 11]. However, the proof of this result is based on quite different methods than ours. It does not use estimates on the SSF; instead the residue theorem is applied to obtain an uniform bound on averaged resolvents. Due to the use of complex analysis it is not clear whether this method can be extended to the case when the single site distribution $\mu$ does not have a density.

Let us sketch the outline of the paper: The next section contains the definition of the SSF and the proofs of Theorems 1 and 2. In Sect. 3 we prove a lemma which is needed to deal with singular coupling constants and complete the proof of the Wegner estimate, Theorem 3.

\section{Bounds on the SSF}

2.1. Definition of the SSF. We define the SSF in three steps. Each of them extends the definition to a larger class of operators. For proofs see, e.g., [5 or 59].

Assume first that $H_{1}, H_{2}$ are selfadjoint, lower-semibounded with purely discrete spectrum. Then the SSF is defined as the difference of the eigenvalue counting functions,

$$
\xi(\lambda):=\#\left\{n \mid \lambda_{n}\left(H_{2}\right) \leq \lambda\right\}-\#\left\{n \mid \lambda_{n}\left(H_{1}\right) \leq \lambda\right\},
$$

where $\lambda_{n}(H)$ enumerates the spectrum of $H$, including multiplicity, in increasing order. Consider now a pair of selfadjoint, lower-semibounded operators such that the difference $H_{2}-H_{1}$ is trace class. Then there is a unique function $\xi$ such that Krein's trace identity

$$
\operatorname{Tr}\left[\rho\left(H_{2}\right)-\rho\left(H_{1}\right)\right]=\int \rho^{\prime}(\lambda) \xi\left(\lambda, H_{2}, H_{1}\right) d \lambda
$$

holds for all $\rho \in \mathcal{C}^{\infty}$ with compactly supported derivative (actually, $\rho$ can be taken to lie in a certain Besov space, see [41]). If the operators have discrete spectrum, this definition of $\xi$ coincides with the previous one. It can be recovered choosing a sequence of switch functions $\rho_{\varepsilon}$ which converges to a step function as $\varepsilon \rightarrow 0$.

Finally, we weaken the trace class assumption on the operator difference. Let $g: \mathbb{R} \rightarrow$ $[0, \infty)$ be a monotone, smooth function such that $g\left(H_{2}\right)-g\left(H_{1}\right)$ is trace class. Assume that $g$ is bounded on the spectra of $H_{1}$ and $H_{2}$. Then the SSF for the operator pair $g\left(H_{1}\right), g\left(H_{2}\right)$ is well defined and we may set

$$
\xi\left(\lambda, H_{2}, H_{1}\right):=\operatorname{sign}\left(g^{\prime}\right) \xi\left(g(\lambda), g\left(H_{2}\right), g\left(H_{1}\right)\right) .
$$

This definition is independent of the choice of $g$. Formula (13) is called the invariance principle. This last definition will be sufficiently general to cover the Schrödinger operators we are considering. In the sequel we will choose $g(x)=e^{-x}$.

Alternatively, the SSF can be defined via the perturbation determinant from scattering theory by

$$
\left.\xi\left(\lambda, H_{1}, H_{2}\right):=\frac{1}{\pi} \lim _{\varepsilon \searrow 0} \arg \operatorname{det}\left[1+\left(H_{1}-H_{2}\right)\left(H_{2}-\lambda-i \varepsilon\right)^{-1}\right)\right]
$$

if $\left(H_{1}-H_{2}\right)\left(H_{2}+i\right)^{-1}$ is trace class. 
2.2. Decay of singular values. Weyl's asymptotic law gives the asymptotic behaviour of the $n^{\text {th }}$ eigenvalue of the Laplacian on an open ball $B$ for large $n$. The following simple lemma provides a robust lower bound, very much in the spirit of Weyl's law, but valid for all $n$ and for general magnetic Schrödinger operators. It is the starting point for our proof of Theorem 1 .

As it costs us nothing in clarity, the lemma will be presented under weaker hypotheses than those described in the introduction.

Lemma 5. Let $H=H_{A}+V=(-i \nabla-A)^{2}+V$ as in the introduction (cf. (1)), except that we now require that $V_{-}$is merely $-\Delta$ bounded with relative bound $\delta<1$. Furthermore, let $H^{\mathcal{U}}$ be the Dirichlet restriction of $H$ to an arbitrary open set $\mathcal{U}$ with finite volume $|\mathcal{U}|$ (also defined via the corresponding quadratic forms). Then, for some constant $C$, the $n^{\text {th }}$ eigenvalue of $H^{\mathcal{U}}$ satisfies

$$
E_{n} \geq \frac{2 \pi(1-\delta) d}{e}\left(\frac{n}{|\mathcal{U}|}\right)^{2 / d}-C \quad \text { for all } n \in \mathbb{N} .
$$

Proof. Since the Dirichlet Sobolev space $H_{0}^{1}(\mathcal{U})$ is a natural subset of $H^{1}\left(\mathbb{R}^{d}\right), V_{-}$is also relatively form bounded w.r.t. $-\Delta^{\mathcal{U}}$, the Dirichlet Laplacian on $\mathcal{U}$ with relative bound $\delta$. The diamagnetic inequality, [48], then implies that $V_{-}$is also relative form bounded w.r.t. to the Dirichlet restriction of $H_{A}$ to $\mathcal{U}$. That is, there exists a $C \in \mathbb{R}$ such that, as quadratic forms,

$$
V_{-} \leq \delta H_{A}+C
$$

In particular, since $V_{+}$is non-negative,

$$
H^{\mathcal{U}} \geq H_{A}-V_{-} \geq(1-\delta) H_{A}-C,
$$

which implies the bound

$$
\begin{aligned}
\operatorname{Tr}\left(e^{-2 t H^{\mathcal{U}}}\right) & \leq e^{2 t C} \operatorname{Tr}\left(e^{-2 t(1-\delta) H_{A}}\right)=e^{2 t C}\left\|e^{-t(1-\delta) H_{A}}\right\|_{\mathrm{HS}}^{2} \\
& =e^{2 t C} \iint_{\mathcal{U} \times \mathcal{U}}\left|e^{-t(1-\delta) H_{A}}(x, y)\right|^{2} d x d y,
\end{aligned}
$$

where $\|\cdot\|_{\text {HS }}$ denotes the Hilbert-Schmidt norm. Again, using the diamagnetic inequality for the Schrödinger semigroup, e.g., [48, 26], one has the pointwise bound $\left|e^{-t(1-\delta) H_{A}}(x, y)\right| \leq e^{t(1-\delta) \Delta^{\mathcal{U}}}(x, y)$. In particular,

$$
\left\|e^{-t(1-\delta) H_{A}}\right\|_{\mathrm{HS}}^{2} \leq\left\|e^{t(1-\delta) \Delta^{\mathcal{U}}}\right\|_{\mathrm{HS}}^{2}=\operatorname{Tr}\left(e^{2 t(1-\delta) \Delta^{\mathcal{U}}}\right) \leq|\mathcal{U}|(8 \pi t(1-\delta))^{-d / 2} .
$$

In the last line we used the fact that the kernel of the Dirichlet semigroup $e^{\beta \Delta^{\mathcal{U}}}$ on the diagonal is bounded by the free kernel, i.e.,

$$
e^{\beta \Delta^{\mathcal{U}}}(x, x) \leq e^{\beta \Delta}(x, x)=(4 \pi \beta)^{-d / 2} \text { for all } \beta>0 \text { and } x \in \mathcal{U},
$$

which follows immediately from the probabilistic representation of the Dirichlet semigroup [3, 47]. Thus

$$
\operatorname{Tr}\left(e^{-2 t H^{\mathcal{U}}}\right) \leq|\mathcal{U}|(8 \pi t(1-\delta))^{-d / 2}
$$

Let $\mathcal{N}^{\mathcal{U}}(E)$ be the number of eigenvalues of $H^{\mathcal{U}}$ smaller or equal to $E$. By Čebyšev's inequality and the above bound, 


$$
\begin{aligned}
\mathcal{N}^{\mathcal{U}}(E) & \leq e^{2 t E} \int_{-\infty}^{E} e^{2 t s} d \mathcal{N}^{\mathcal{U}}(s) \leq e^{2 t E} \operatorname{Tr}\left(e^{-2 t H^{\mathcal{U}}}\right) \\
& \leq|\mathcal{U}|(8 \pi(1-\delta))^{-d / 2} t^{-d / 2} e^{2 t(E+C)}=|\mathcal{U}|\left(\frac{e(E+C)}{2 \pi(1-\delta) d}\right)^{d / 2}
\end{aligned}
$$

where, in the last equality, we choose $t:=\frac{d}{4(E+C)}$. Since $n \leq \mathcal{N}^{\mathcal{U}}\left(E_{n}\right)$, this, in turn, implies the lower bound

$$
E_{n} \geq \frac{2 \pi(1-\delta) d}{e}\left(\frac{n}{|\mathcal{U}|}\right)^{2 / d}-C
$$

on the eigenvalues.

Proof of Theorem 1. We give the proof for $V_{\mathrm{eff}}$, the adaption to $V_{\mathrm{eff}}^{l}$ requires only minor changes. We will use the symbols $c$ and $C$ for constants that vary from line to line; however, their dependence on $H_{1}$ and $H_{2}$ will always be as stated in the theorem.

Without loss of generality, we can assume that the origin is contained in the support of $u$. We will estimate the $n^{\text {th }}$ singular value by Dirichlet decoupling at an $n$-dependent radius $R$. To this end, let $R$ be sufficiently large that $\operatorname{supp}(u)$ is contained strictly inside the ball of radius $R$ centered at the origin, which we will denote by $B_{R}$.

Let $H_{j}^{R}(j=1$ or 2$)$ be the Dirichlet restriction of $H_{j}$ to the $B_{R}$, and let

$$
A_{R}:=e^{-H_{2}^{R}}-e^{-H_{1}^{R}} \quad \text { and } \quad D_{R}:=V_{\mathrm{eff}}-A_{R} .
$$

As any Kato-class potential is relatively form bounded with respect to the Laplacian with relative bound zero, we may apply Lemma 5 to deduce that $\mu_{n}\left(e^{-H_{j}^{n}}\right) \leq C$ $\exp \left(-c n^{2 / d} R^{-2}\right)$ for both $j=1$ and $j=2$. Since $A_{R}$ is the difference of two $n$ onnegative operators by the min-max theorem its singular values obey the same type of bound:

$$
\mu_{n}\left(A_{R}\right) \leq C \exp \left(-c n^{2 / d} R^{-2}\right)
$$

If $D_{n}$ is bounded, then $\mu_{n}\left(V_{\text {eff }}\right) \leq \mu_{n}\left(A_{R}\right)+\left\|D_{n}\right\|$. We now proceed to estimate the norm of $D_{n}$ by using the Feynman-Kac-Itô formula for magnetic Schrödinger semigroups with Dirichlet boundary conditions, see [6, 48].

Let $\mathbf{E}_{x}$ and $\mathbf{P}_{x}$ denote the expectation and probability for a Brownian motion, $b_{t}$ starting at $x$. If $\tau_{R}=\inf \left\{t>0 \mid b_{t} \notin B_{R}\right\}$ denotes the exit time from the ball $B_{R}$ and $\tau_{n}:=\tau_{R_{n}}$, then

$$
\left(D_{n} f\right)(x)=\mathbf{E}_{x}\left[e^{-i S_{A}(b)}\left(e^{-\int_{0}^{1}(V+u)\left(b_{s}\right) d s}-e^{-\int_{0}^{1} V\left(b_{s}\right) d s}\right) \chi_{\left\{\tau_{n} \leq 1\right\}}(b) f\left(b_{1}\right)\right],
$$

where $S_{A}^{t}$ is real valued stochastic process corresponding to the purely magnetic part of the Schrödinger operator. To be precise, one has to fix a suitable gauge, e.g., Coulomb gauge, i.e., $\operatorname{div} A=0$, for this and then use gauge invariance for the general case, see [38].

By taking the modulus and using the triangle inequality, one sees that the magnetic vector potential drops out:

$$
\left|D_{n} f\right|(x) \leq \mathbf{E}_{x}\left[e^{-\int_{0}^{1} V\left(b_{s}\right) d s}\left|e^{-\int_{0}^{1} u\left(b_{s}\right) d s}-1\right| \chi_{\left\{\tau_{n} \leq 1\right\}}(b)\left|f\left(b_{1}\right)\right|\right] .
$$


Moreover, only Brownian paths which both visit supp $u$ and leave $B_{R_{n}}$ within one unit of time contribute to the expectation. Thus if $\tau_{u}$ is the hitting time for $\operatorname{supp}(u)$ and $\mathcal{B}=\left\{\tau_{R} \leq 1, \tau_{u} \leq 1\right\}$, where we abbreviate $R=R_{n}$, then

$$
\left|D_{n} f\right|(x) \leq \mathbf{E}_{x}\left[e^{-\int_{0}^{1} V\left(b_{s}\right) d s}\left|e^{-\int_{0}^{1} u\left(b_{s}\right) d s}-1\right| \chi_{\mathcal{B}}(b)\left|f\left(b_{1}\right)\right|\right],
$$

so, applying Hölder's inequality,

$$
\begin{aligned}
\left|D_{n} f\right|(x) \leq( & \left.\mathbf{E}_{x}\left[e^{-8 \int_{0}^{1} V\left(b_{s}\right) d s}\right]\right)^{1 / 8}\left(\mathbf{E}_{x}\left[\left|e^{-\int_{0}^{1} u\left(b_{s}\right) d s}-1\right|^{8}\right)^{1 / 8}\right. \\
& \times\left(\mathbf{E}_{x}\left[\chi_{\mathcal{B}}(b)\right]\right)^{1 / 4}\left(\mathbf{E}_{x}\left[\left|f\left(b_{1}\right)\right|^{2}\right]\right)^{1 / 2} .
\end{aligned}
$$

By Kashminskii's lemma, the Kato-class condition on $V_{-}$and $u_{-}$implies that the first two terms are bounded uniformly in $x,[2,49]$.

Levy's inequality combined with elementary estimates imply $\mathbf{P}_{x=0}\left\{\tau_{R} \leq 1\right\} \leq$ $2 \mathbf{P}_{x=0}\left\{\left|b_{1}\right| \geq R\right\} \leq C e^{-R^{2} / 4}$. As any path in $\mathcal{B}$ must cover the distance $r$ between supp $u$ and the complement of the ball $B_{R}$, we can deduce that $\mathbf{P}_{x}(\mathcal{B}) \leq C e^{-r^{2} / 4} \leq$ $C e^{-R^{2} / 8}$, where we chose without loss of generality $r \geq R / \sqrt{2}$. Thus

$$
\left|D_{n} f\right|(x) \leq C e^{-R^{2} / 32}\left\{\mathbf{E}_{x}\left|f\left(b_{1}\right)\right|^{2}\right\}^{1 / 2}=C e^{-R^{2} / 32}\left\{\left(e^{\Delta}|f|^{2}\right)(x)\right\}^{1 / 2},
$$

in particular, using the fact that $e^{\Delta}$ is an $L^{1}$ contraction,

$$
\left\|D_{n} f\right\|_{2} \leq C e^{-R^{2} / 32}\left\|\left(e^{\Delta}|f|^{2}\right)\right\|_{1}^{1 / 2} \leq C e^{-R^{2} / 32}\left\|f^{2}\right\|_{1}^{1 / 2}=C e^{-R^{2} / 32}\|f\|_{2} .
$$

To balance the two bounds obtained for $\mu_{n}\left(A_{R}\right)$ and $\left\|D_{n}\right\|$ one chooses $R_{n}:=n^{1 / 2 d}$, which leads to (2).

\subsection{Singular value decay implies SSF estimate.}

Proof of Theorem 2. Let $F_{t}$ and the two Schrödinger operators $H_{2}=H_{1}+u$ be as in the theorem. Using the invariance principle and a change of variables, we have

$$
\begin{aligned}
\int_{-\infty}^{T} F\left(\left|\xi\left(\lambda, H_{2}, H_{1}\right)\right|\right) d \lambda & =\int_{-\infty}^{T} F\left(\left|\xi\left(e^{-\lambda}, e^{-H_{2}}, e^{-H_{1}}\right)\right|\right) d \lambda \\
& \leq e^{T} \int_{e^{-T}}^{\infty} F\left(\left|\xi\left(s, e^{-H_{2}}, e^{-H_{1}}\right)\right|\right) d s
\end{aligned}
$$

By an estimate of Hundertmark and Simon [25], the integral on the RHS is bounded by

$$
\begin{aligned}
\int_{-\infty}^{\infty} F\left(\left|\xi\left(s, e^{-H_{2}}, e^{-H_{1}}\right)\right|\right) d s & \leq \sum_{n=1}^{\infty} \mu_{n}\left(V_{\mathrm{eff}}\right)(F(n)-F(n-1)) \\
& \leq \sum_{n=1}^{\infty} \mu_{n}\left(V_{\mathrm{eff}}\right) \int_{n-1}^{n}\left(e^{t s^{1 / d}}-1\right) d s \\
& \leq C \sum_{n=1}^{\infty} e^{(t-c) n^{1 / d}}
\end{aligned}
$$

which is finite, if we chose $t$ smaller than the constant $c$ from Theorem 1 . This proves (4). 
To prove (5), we dualize the bound (4) with the help of Young's inequality for an appropriate pair of functions. Note that $F_{t}$ is non-negative, convex with $F_{t}^{\prime}(0)=0$ and hence its Legendre transform $G$ is well defined and satisfies

$$
G(y):=\sup _{x \geq 0}\{x y-F(x)\} \leq y\left(\frac{\log (1+y)}{t}\right)^{d} \text { for all } y \geq 0 .
$$

Thus, by the very definition of $G$, Young's inequality holds: $y x \leq F(x)+G(y)$. So, with $b=\sup \operatorname{supp}(f)$,

$$
\int f(\lambda) \xi(\lambda) d \lambda \leq \int_{-\infty}^{b} F(|\xi(\lambda)|) d \lambda+\int G(|f(\lambda)|) d \lambda
$$

Using the estimate (4), the first integral is bounded by $K_{1} e^{b}$. For the second integral in (17), we estimate

$$
\int G(|f(\lambda)|) d \lambda \leq \int|f(\lambda)|\left(\frac{\log (1+|f(\lambda)|)}{t}\right)^{d} d \lambda \leq t^{-d}\left|\log \left(1+\|f\|_{\infty}\right)\right|^{d}\|f\|_{1} .
$$

This finishes the proof of Theorem 2 .

\section{Proof of the Wegner Estimate}

3.1. A partial integration formula for singular distributions. The main new idea to deal with single site distributions that are not absolutely continuous, is the following simple

Lemma 6. Let $\mu$ be a probability measure with support in $(a, b))($ or $(a, \infty)$, if its support is unbounded from above) and $\phi \in C^{1}(\mathbb{R})$ be non-decreasing and bounded. Then for any $\varepsilon>0$,

$$
\int_{\mathbb{R}}[\phi(\lambda+\varepsilon)-\phi(\lambda)] d \mu(\lambda) \leq s(\mu, \varepsilon) \cdot[\phi(b+\varepsilon)-\phi(a)],
$$

where $s(\mu, \varepsilon)$, the modulus of continuity of $\mu$, is defined in (9). (If $b=\infty, \phi(b+\epsilon)$ means $\lim _{x \rightarrow \infty} \phi(x)$, which exists by the properties of $\left.\phi.\right)$

Proof. The proof of this lemma follows immediately from the well-known integrationby-parts formula for Stieltjes integrals. We include it for the convenience of the reader. We write $d \mu=d M$, where $M$ is the distribution function of $\mu$. In the following, all integrals are defined as Stieltjes integrals. Shifting variables and using that $M$ is constant outside of $[a, b]$ gives

$$
\int[\phi(\lambda+\varepsilon)-\phi(\lambda)] d \mu(\lambda)=\int_{a}^{b+\varepsilon} \phi(\lambda) d[M(\lambda-\varepsilon)-M(\lambda)] .
$$

Integrating by parts gives

$$
\begin{aligned}
\int[\phi(\lambda+\varepsilon)-\phi(\lambda)] d \mu(\lambda)= & {[\phi(\lambda)[M(\lambda-\varepsilon)-M(\lambda)]]_{a}^{b+\varepsilon} } \\
& -\int_{a}^{b+\varepsilon} \phi^{\prime}(\lambda)[M(\lambda-\varepsilon)-M(\lambda)] d \lambda .
\end{aligned}
$$


The first term is zero, since $M$ is constant outside of $[a, b]$ (in case $b=\infty$, one uses boundedness of $\phi$ and $\left.\lim _{\lambda \rightarrow \infty}[M(\lambda-\varepsilon)-M(\lambda)]=0\right)$. The second term is bounded by

$$
\begin{aligned}
\int_{a}^{b+\varepsilon} \phi^{\prime}(\lambda)[M(\lambda)-M(\lambda-\varepsilon)] d \lambda & \leq \sup _{\lambda}[M(\lambda)-M(\lambda-\varepsilon)] \cdot \int_{a}^{b+\varepsilon} \phi^{\prime}(\lambda) d \lambda \\
& \leq s(\mu, \varepsilon) \cdot(\phi(b+\varepsilon)-\phi(a)),
\end{aligned}
$$

since $\phi^{\prime} \geq 0$.

3.2. Proof of the Wegner estimate. Let $\rho$ be a switch function adapted to the interval $[E-\varepsilon, E+\varepsilon]$; see the discussion preceding (7). Then

$$
\chi_{[E-\varepsilon, E+\varepsilon]}(x) \leq \rho(x+2 \varepsilon)-\rho(x-2 \varepsilon) .
$$

We may assume without loss of generality $\sum_{k} u(\cdot-k) \geq 1$. By the mini-max principle for eigenvalues, we conclude

$$
\operatorname{Tr}\left[\rho\left(H_{\omega}^{l}+\varepsilon\right)\right] \leq \operatorname{Tr}\left[\rho\left(H_{\omega}^{l}+\varepsilon \sum_{k} u(\cdot-k)\right)\right] .
$$

Assume without loss of generality that $l \in \mathbb{N}$. Then $\Lambda_{l}$ is decomposed in $L:=l^{d}$ unit cubes. We enumerate the lattice sites in $\Lambda_{l}$ by $k:\{1, \ldots, L\} \rightarrow \tilde{\Lambda}=\Lambda \cap \mathbb{Z}^{d}, n \mapsto k(n)$ and set

$$
W_{0} \equiv 0, \quad W_{n}=\sum_{m=1}^{n} u(\cdot-k(m)), \quad n=1,2, \ldots, L
$$

Thus

$$
\begin{aligned}
\mathbb{E}\left\{\operatorname{Tr}\left[\chi_{[E-\varepsilon, E+\varepsilon]}\left(H_{\omega}^{l}\right)\right]\right\} & \leq \mathbb{E}\left\{\operatorname{Tr}\left[\rho\left(H_{\omega}^{l}+2 \varepsilon\right)-\rho\left(H_{\omega}^{l}-2 \varepsilon\right)\right]\right\} \\
& \leq \mathbb{E}\left\{\operatorname{Tr}\left[\rho\left(H_{\omega}^{l}+2 \varepsilon\right)-\rho\left(H_{\omega}^{l}+2 \varepsilon-4 \varepsilon W_{L}\right)\right]\right\} \\
& \leq \mathbb{E}\left\{\sum_{n=1}^{L} \operatorname{Tr}\left[\rho\left(H_{\omega}^{l}+2 \varepsilon-4 \varepsilon W_{n-1}\right)-\rho\left(H_{\omega}^{l}+2 \varepsilon-4 \varepsilon W_{n}\right)\right]\right\} .
\end{aligned}
$$

We fix $n \in\{1, \ldots, L\}$, denote $k_{0}=k(n)$,

$$
\omega^{\perp}:=\left\{\omega_{k}^{\perp}\right\}_{k \in \tilde{\Lambda}}, \quad \omega_{k}^{\perp}:= \begin{cases}0 & \text { if } k=k_{0}, \\ \omega_{k} & \text { if } k \neq k_{0},\end{cases}
$$

and set

$$
\phi\left(\omega_{k_{0}}\right)=\operatorname{Tr}\left[\rho\left(H_{\omega^{\perp}}^{l}-2 \varepsilon+4 \varepsilon W_{n-1}+\omega_{k_{0}} u\left(\cdot-k_{0}\right)\right)\right], \quad \omega_{n} \in \mathbb{R} .
$$

The function $\phi$ is continuously differentiable, monotone increasing and bounded. By definition of $\phi$,

$$
\begin{aligned}
\mathbb{E} & \left.\left\{\operatorname{Tr}\left[\rho\left(H_{\omega}^{l}+2 \varepsilon-4 \varepsilon W_{n}\right)\right)-\rho\left(H_{\omega}^{l}+2 \varepsilon-4 \varepsilon W_{n+1}\right)\right]\right\} \\
\leq & \mathbb{E}\left\{\int\left[\phi\left(\omega_{k_{0}}+2 \varepsilon\right)-\phi\left(\omega_{k_{0}}\right)\right] d \mu\left(\omega_{k_{0}}\right)\right\} .
\end{aligned}
$$


Let $a=\inf \operatorname{supp}(\mu)-1$ and $b=\sup \operatorname{supp}(\mu)+1$. Using Lemma 6 and the Krein trace identity (12) together with the second part of Theorem 2 , we have

$$
\begin{aligned}
\int\left[\phi\left(\omega_{k_{0}}+2 \varepsilon\right)-\phi\left(\omega_{k_{0}}\right)\right] d \mu\left(\omega_{k_{0}}\right) & \leq s(\mu, 2 \varepsilon)[\phi(b+2 \varepsilon)-\phi(a)] \\
& \leq C_{E} s(\mu, 2 \varepsilon)(\log (1 / \varepsilon))^{d},
\end{aligned}
$$

which implies that (18) is bounded by

$$
C_{E} \sum_{n=1}^{L} s(\mu, 2 \varepsilon)(\log (1 / \varepsilon))^{d} \leq C_{E} s(\mu, 2 \varepsilon)(\log (1 / \varepsilon))^{d} l^{d} .
$$

Note that we apply Theorem 2 successively $L$ times. However, the constant $C_{E}$ depends only on the diameter of $u$ and a local norm of the negative part of the background potential. For this local norm there exist an uniform estimate independent of $\Lambda_{l}$ and the configuration of the coupling constants $\omega_{k}, k \neq k_{0}$.

Acknowledgements. Stimulating discussions with W. Kirsch, V. Kostrykin, G. Stolz and S. Warzel are gratefully acknowledged. P.S. acknowledges the kind invitation to the University of Tokyo where part of this work started. D.H and I.V. would like to thank the Department of Mathematics at CalTech, especially B. Simon and C. Galvez, for their warm hospitality. We thank the following institutions for financial support: the National Science Foundation for their support under grants DMS-0400940 (D.H.) and DMS-0401277 (R.K.), the Sloan Foundation (R.K.), the JSPS through Grant-in-Aid for Scientific Research (C) 13640155 (S.N.), the DFG through the SFB 393 (P.S.) and grants Ve 253/1 and /2 within the Emmg-Noether Programme (I.V.).

See also www.arxiv.org/math-ph/0412078 or www.ma.utexas.edu/mp_arc/c/04/04-423.pdf for an earlier version of this paper.

\section{References}

1. Aizenman, M., Elgart, A., Naboko, S., Schenker, J.H., Stolz G.: Moment Analysis for Localization in Random Schrödinger Operators. http://arxiv.org/list/math-ph/0308023, 2003, Invent. Math. online first (2005)

2. Aizenman, M., Simon, B.: Brownian motion and Harnack inequality for Schrödinger operators. Comm. Pure Appl. Math. 35(2), 209-273 (1982)

3. Bass, R.F.: Probabilistic techniques in analysis. In: Probability and its Applications (New York), New York: Springer-Verlag, 1995

4. Barbaroux, J.-M., Combes, J.-M., Hislop, P.D.: Localization near band edges for random Schrödinger operators. Helv. Phys. Acta 70(1-2), 16-43 (1997)

5. Birman, M.Š., Yafaev, D.: The spectral shift function. The work of M.G. Krein and its further development. St. Petersburg Math. J. 4, 833-870 (1993)

6. Broderix, K., Hundertmark, D., Leschke, H.: Continuity properties of Schrödinger semigroups with magnetic fields. Rev. Math. Phys. 12(2), 181-225 (2000)

7. van Casteren, J.A., Demuth, M.: On differences of heat semigroups. Ann. Sci. Univ. ClermontFerrand II Probab. Appl. (8), 119-147 (1990)

8. van Casteren, J.A., Demuth, M.: Some results in stochastic spectral analysis. In: Partial differential equations (Han-sur-Lesse, 1993), Volume 82 of Math. Res., Berlin: Akademie-Verlag, 1994, pp. 56-81

9. van Casteren, J., Demuth, M., Stollmann, P., Stolz, G.: Trace norm estimates for products of integral operators and diffusion semigroups. Integral Equations Operator Theory 23(2), 145-153 (1995)

10. Chahrour, A.: On the spectrum of the Schrödinger operator with periodic surface potential. Lett. Math. Phys. 52(3), 197-209 (2000)

11. Combes, J.-M., Hislop, P.: Localization for some continuous, random Hamiltionians in d-dimensions. J. Funct. Anal. 124, 149-180 (1994)

12. Combes, J.-M., Hislop, P.D., Klopp, F.: Hölder continuity of the integrated density of states for some random operators at all energies. Int. Math. Res. Not. (4), 179-209 (2003) 
13. Combes, J.-M., Hislop, P.D., Klopp, F., Nakamura, S.: The Wegner estimate and the integrated density of states for some random operators. Proc. Indian Acad. Sci. Math. Sci. 112(1), 31-53 (2002)

14. Combes, J.-M., Hislop, P.D., Nakamura, S.: The $L^{p}$-theory of the spectral shift function, the Wegner estimate, and the integrated density of states for some random Schrödinger operators. Commun. Math. Phys. 218, 113-130 (2001)

15. Cycon, H.L., Froese, R.G., Kirsch, W., Simon, B.: Schrödinger operators with application to quantum mechanics and global geometry. Texts and Monographs in Physics. Springer Study Edition. Berlin: Springer-Verlag, 1987

16. Deift, P., Simon, B.: On the decoupling of finite singularities from the question of asymptotic completeness in two body quantum systems. J. Funct. Anal. 23(3), 218-238 (1976)

17. Demuth, M.: On scattering of diffusion process generators. Lett. Math. Phys. 4(5), 417-424 (1980)

18. Demuth, M.: Traces and trace norms for semigroup differences. In: Mathematical results in quantum mechanics (Prague, 1998), Volume 108 of Oper. Theory Adv. Appl., Basel: Birkhäuser, 1999, pp. 209-219

19. Demuth, M., Kirsch, W., McGillivray, I.: Schrödinger operators-geometric estimates in terms of the occupation time. Commun. Part. Diff. Eq. 20, 37-57 (1995)

20. Geisler, R., Kostrykin, V., Schrader, R.: Concavity properties of Krel̆n's spectral shift function. Rev. Math. Phys., 7(2), 161-181 (1995)

21. Germinet, F., Klein, A.: A characterization of the Anderson metal-insulator transport transition. Duke Math. J. 124, no. 2, 309-350 (2001)

22. Gesztesy, F., Makarov, K.A., Motovilov, A.K.: Monotonicity and concavity properties of the spectral shift function. In: Stochastic processes, physics and geometry: new interplays, II (Leipzig, 1999), Volume 29 of CMS Conf. Proc., Providence, RI: Amer. Math. Soc., 2000, pp. 207-222

23. Hislop, P.D.: Private communication

24. Hislop, P.D., Klopp, F.: The integrated density of states for some random operators with nonsign definite potentials. J. Funct. Anal. 195(1), 12-47 (2002)

25. Hundertmark, D., Simon, B.: An optimal $L^{p}$-bound on the Krein spectral shift function. J. Anal. Math. 87, 199-208 (2002)

26. Hundertmark, D., Simon, B.: A diamagnetic inequality for semigroup differences. J. Pure Appl. Math. (Crelle) 571, 107-130 (2004)

27. Hupfer, T., Leschke, H., Müller, P., Warzel, S.: The absolute continuity of the integrated density of states for magnetic Schrödinger operators with certain unbounded random potentials. Commun. Math. Phys. 221(2), 229-254 (2001)

28. Kirsch, W.: Small perturbations and the eigenvalues of the Laplacian on large bounded domains. Proc. Am. Math. Soc. 101, 509-512 (1987)

29. Kirsch, W.: The stability of the density of states of Schrödinger operator under very small perturbations. Int. Equations Operator Th. 12, 383-391 (1989)

30. Kirsch, W.: Wegner estimates and Anderson localization for alloy-type potentials. Math. Z. 221, 507-512 (1996)

31. Kirsch, W., Stollmann, P., Stolz, G.: Anderson localization for random Schrödinger operators with long range interactions. Commun. Math. Phys. 195(3), 495-507 (1998)

32. Kirsch, W., Stollmann, P., Stolz, G.: Localization for random perturbations of periodic Schrödinger operators. In: Random Oper. Stoch. Eqs. 6(3), 241-268 (1998) (Available at http://www.ma.ut exas.edu/mp_arc)

33. Kirsch, W., Veselić, I.: Wegner estimate for sparse and other generalized alloy type potentials. Proc. Indian Acad. Sci. Math. Sci. 112(1), 131-146 (2002)

34. Kostrykin, V.: The spectral shift function and its applications to random Schrödinger operators. Habilitation Thesis, RWTH Aachen, 1999

35. Kostrykin, V.: Concavity of eigenvalue sums and the spectral shift function. J. Funct. Anal. 176(1), $100-114(2000)$

36. Kostrykin, V., Schrader, R.: Regularity of the surface density of states. J. Funct. Anal. 187(1), 227$246(2001)$

37. Kotani, S., Simon, B.: Localization in general one-dimensional random systems. II. Continuum Schrödinger operators. Commun. Math. Phys. 112(1), 103-119 (1987)

38. Leinfelder, H.: Gauge invariance of Schrödinger operators and related spectral properties. J. Operator Theory 9(1), 163-179 (1983)

39. Melgaard, M., Rozenblum, G.: Eigenvalue asymptotics for weakly perturbed Dirac and Schrödinger operators with constant magnetic fields of full rank. Comm. Part. Differ. Eqs. 28(3-4), 697-736 (2003)

40. Nakamura, S.: Spectral shift function for trapping energies in the semiclassical limit. Commun. Math. Phys. 208(1), 173-193 (1999) 
41. Peller, V.V.: Hankel operators in the perturbation theory of unbounded selfadjoint operators. In: Analysis and partial differential equations, Volume 122 of Lecture Notes in Pure and Appl. Math., New York: Dekker, 1990, pp. 529-544

42. Pushnitski, A.: Estimates for the spectral shift function of the polyharmonic operator. J. Math. Phys. 40(11), 5578-5592 (1999)

43. Pushnitskiĭ, A.B.: Spectral shift function of the Schrödinger operator in the large coupling constant limit. Comm. Part. Differ. Eqs. 25(3-4), 703-736 (2000)

44. Pushnitskiı̆, A.B., Ruzhansky, M.: Spectral shift function of the Schrödinger operator in the large coupling constant limit. II. Positive perturbations. Comm. Part. Differ. Eq. 27(7-8), 1373-1405 (2002)

45. Raikov, G.D., Warzel, S.: Quasi-classical versus non-classical spectral asymptotics for magnetic Schrödinger operators with decreasing electric potentials. Rev. Math. Phys. 14(10), 1051-1072 (2002)

46. Safronov, O.: Spectral shift function in the large coupling constant limit. J. Funct. Anal. 182(1), $151-169(2001)$

47. Simon B.: Classical boundary conditions as a technical tool in modern mathematical physics. Adv. in Math. 30(3), 268-281 (1978)

48. Simon, B.: Functional integration and quantum physics. Volume 86 of Pure and Applied Mathematics. New York: Academic Press Inc., 1979

49. Simon, B.: Schrödinger semigroups. Bull. Amer. Math. Soc. (N.S.) 7(3), 447-526 (1982); Erratum:"Schrödinger semigroups". Bull. Amer. Math. Soc. (N.S.) 11(2), 426 (1984)

50. Sobolev, A.: Efficient bounds for the spectral shift function. Ann. Inst. Henri Poincaré 58, 55-83 (1993)

51. Stollmann, P.: Scattering by obstacles of finite capacity. J. Funct. Anal. 121(2), 416-425 (1994)

52. Stollmann, P.: Trace ideal properties of perturbed Dirichlet semigroups. In: Mathematical results in quantum mechanics (Blossin, 1993), Volume 70 of Oper. Theory Adv. Appl., Basel: Birkhäuser, 1994, pp. 153-158

53. Stollmann, P.: A convergence theorem for Dirichlet forms with applications to boundary value problems with varying domains. Math. Z. 219, 275-287 (1995)

54. Stollmann, P.: Wegner estimates and localization for continuum Anderson models with some singular distributions. Arch. Math. (Basel) 75(4), 307-311 (2000)

55. Stollmann, P.: Caught by disorder: A Course on Bound States in Random Media. Volume 20 of Progress in Mathematical Physics. Basel-Boston: Birkhäuser, 2001

56. Veselić, I.: Wegner estimate and the density of states of some indefinite alloy type Schrödinger operators. Lett. Math. Phys. 59(3), 199-214 (2002)

57. Veselić, I.: Integrated density of states and Wegner estimates for random Schrödinger operators. In: Villegas-Blas, C., del Rio, R. (eds) Schrödinger operators (Universidad Nacional Autonoma de Mexico, 2001), Volume 340 of Contemp. Math., Providence, RI: Amer. Math. Soc., 2004, pp. 98-184

58. Wegner, F.: Bounds on the DOS in disordered systems. Z. Phys. B 44, 9-15 (1981)

59. Yafaev, D.: Mathematical Scattering Theory. Translations of Mathematical Monographs, 105. Providence, RI: Amer. Math. Soc., 1992. [Russian original: Izdatel'stvo Sankt-Peterburgskogo Universiteta, St. Petersburg, 1994]

Communicated by B. Simon 